\title{
Corrigendum
}

\section{Corrigendum to "The Efficacy and Safety of Chinese Herbal Decoction in Type 2 Diabetes: A 5-Year Retrospective Study"}

\author{
Jiaxing Tian, ${ }^{1,2}$ Fengmei Lian, ${ }^{2}$ Xiaotong Yu, ${ }^{1}$ Yashan Cui, ${ }^{3}$ Tianyu Zhao, ${ }^{4}$ \\ Yang Cao, ${ }^{1}$ and Xiaolin Tong ${ }^{2}$ \\ ${ }^{1}$ Beijing University of Chinese Medicine, Beijing 100029, China \\ ${ }^{2}$ Guanganmen Hospital, China Academy of Chinese Medical Sciences, Beijing 100053, China \\ ${ }^{3}$ Xiyuan Hospital, China Academy of Chinese Medical Sciences, Beijing 100091, China \\ ${ }^{4}$ First Teaching Hospital, Tianjin University of Traditional Chinese Medicine, Tianjin 300192, China \\ Correspondence should be addressed to Xiaolin Tong; xiaolintong66@sina.com \\ Received 11 January 2017; Accepted 17 January 2017; Published 15 February 2017 \\ Copyright (c) 2017 Jiaxing Tian et al. This is an open access article distributed under the Creative Commons Attribution License, \\ which permits unrestricted use, distribution, and reproduction in any medium, provided the original work is properly cited.
}

In the article titled "The Efficacy and Safety of Chinese Herbal Decoction in Type 2 Diabetes: A 5-Year Retrospective Study" [1], there was an error in the order of the first and second affiliations. The corrected affiliation list is shown above.

\section{References}

[1] J. Tian, F. Lian, X. Yu et al., “The efficacy and safety of Chinese herbal decoction in type 2 diabetes: a 5-year retrospective study," Evidence-Based Complementary and Alternative Medicine, vol. 2016, Article ID 5473015, 8 pages, 2016. 


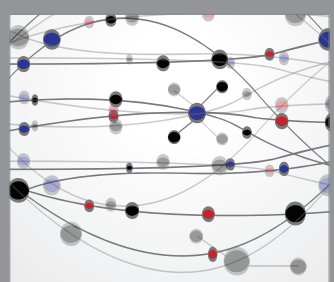

The Scientific World Journal
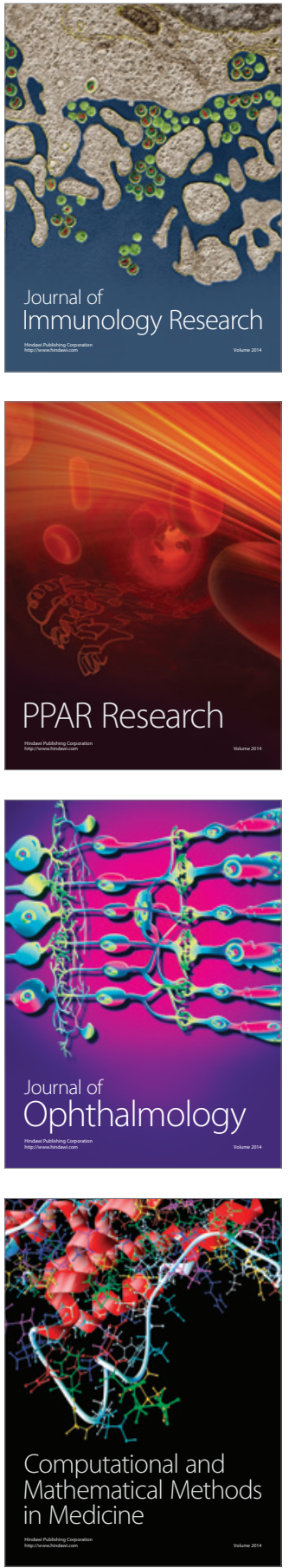

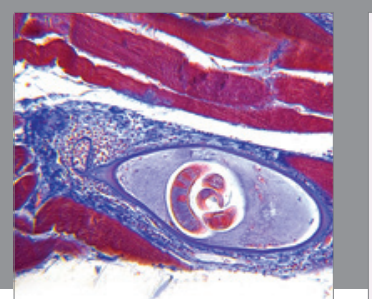

Gastroenterology Research and Practice
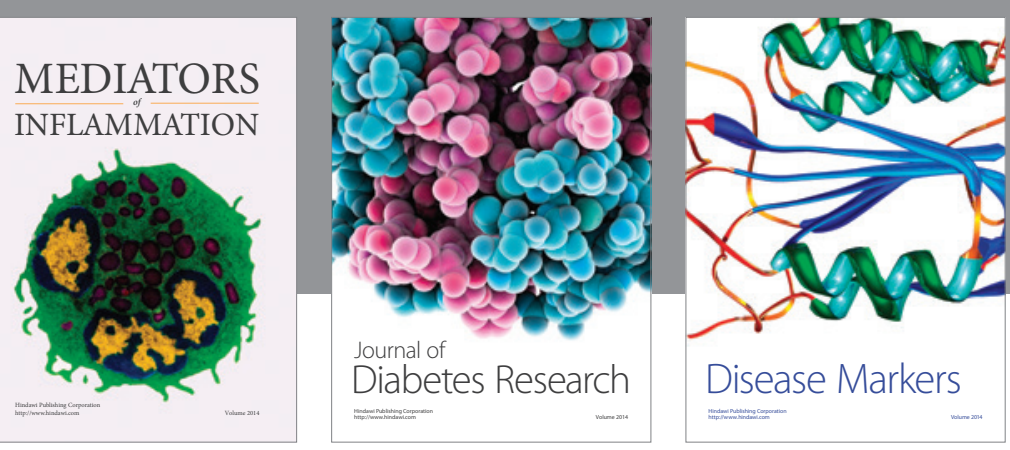

Disease Markers

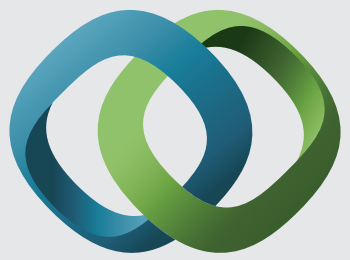

\section{Hindawi}

Submit your manuscripts at

https://www.hindawi.com
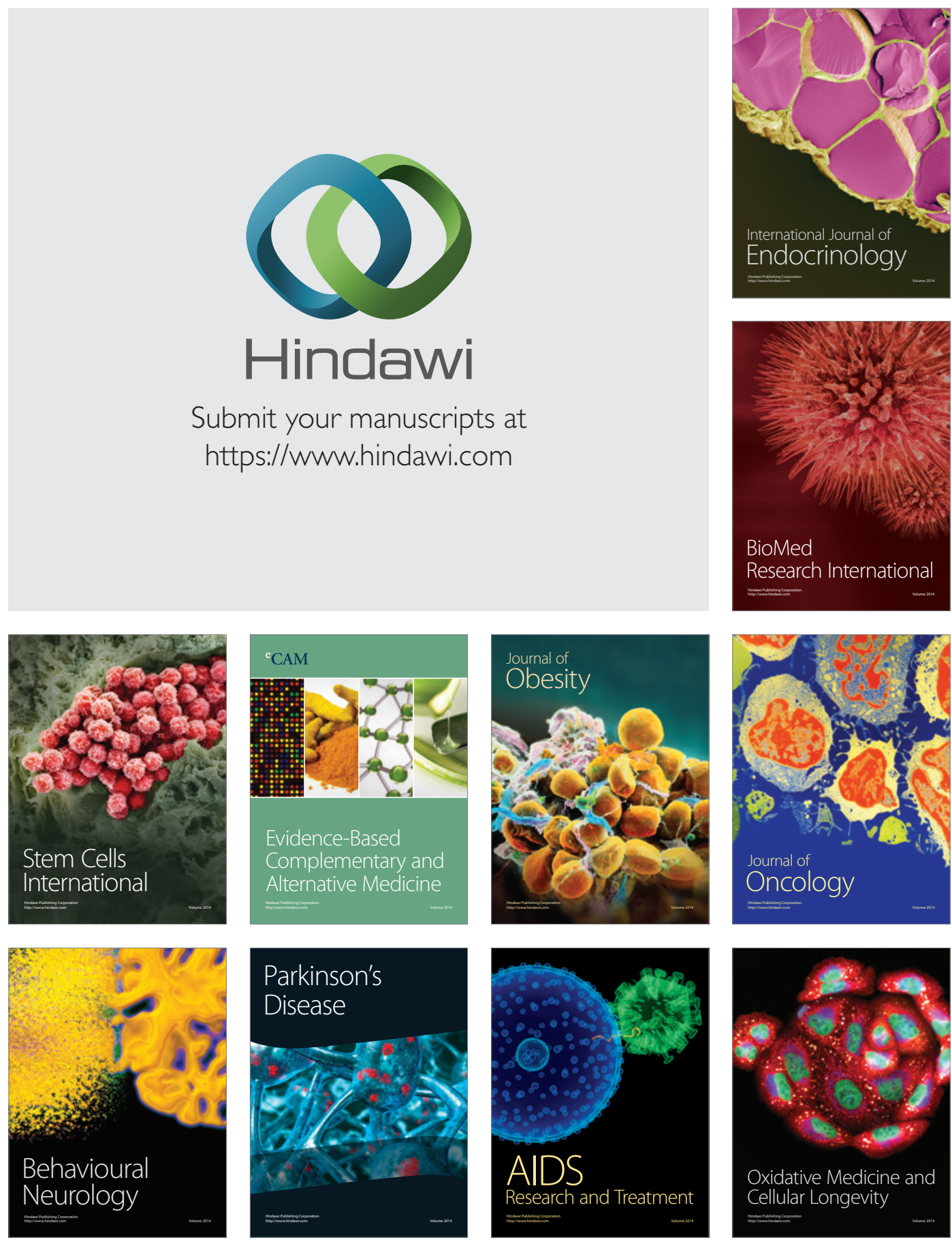